UVX 2012, 02003 (2013)

DOI: $10.1051 /$ uvx/201302003

(C) Owned by the authors, published by EDP Sciences, 2013

\title{
Fabrication of microcraters on silicon substrate by UV nanosecond photonic nanojets from microspheres
}

\author{
K.L.N. Deepak ${ }^{1}$, D. Grojo ${ }^{1}$, L. Charmasson ${ }^{1}$, P. Delaporte ${ }^{1}$, O. Utéza ${ }^{1}$, \\ M. Dussauze ${ }^{2}$ and E. Fargin ${ }^{3}$ \\ ${ }^{1}$ LP3, UMR 7341 CNRS - Aix-Marseille Université, Case 917, 163 avenue de Luminy, \\ 13288 Marseille Cedex 09, France \\ 2 ISM, UMR 5255 CNRS - Université Bordeaux I, Bâtiment A12, 351 bld. de la libération, \\ 33405 Talence Cedex, France \\ ${ }^{3}$ ICMCB, UPR 9048 CNRS, 87 avenue du Docteur Schweitzer, 33608 Pessac Cedex, France
}

\begin{abstract}
We report briefly on the fabrication of arrays of nano- and micro-craters on silicon ( $\mathrm{Si}$ ) substrates using films of $\mathrm{C} 18$ functionalized $1 \mu \mathrm{m}$ silica $\left(\mathrm{SiO}_{2}\right)$ particles deposited by the Langmuir-Blodgett (LB) technique. The films are illuminated with UV nanosecond laser pulses. Local substrate ablation is observed at low fluences well below the damage threshold of silicon $\left(1 \mathrm{~J} / \mathrm{cm}^{2}\right)$. The result is quantitatively explained through FDTD simulations of the micro lensing effect associated with the microspheres. Change in feature size and morphology with number of shots and laser fluence is investigated. In multi-shot irradiation experiments, the produced arrays of well-defined craters rely on the combination of direct laser ablation and laser surface cleaning or annealing as evidenced by Atomic Force Microscopy (AFM) technique.
\end{abstract}

\section{INTRODUCTION}

Fabrication of structures in different materials using conventional optical lithography is limited by diffraction limit of the focusing lens [1]. Fabrication of structures below wavelength used for machining has its applications in areas related to optical devices, spectroscopy, optical microscopy and data storage $[2,3]$. Since the diffraction limit of the focusing light waves does not allow to fabricate structures much smaller than light wavelength, photonic nanojets have emerged as an alternate solution to address the problem. Optical near-field enhancement underneath a monolayer of nanospheres can lead to laser spots with size well below the wavelength and the structures obtained thereby have features at the nanometer scale [2-4]. This technology involves focusing of light waves underneath the surface of a monolayer of particles to fabricate arrays of structures with micro- and nanometer size by reaching locally the fluence above the modification threshold of the material of interest [5-7].

\section{PREPARATION OF SUBSTRATES}

We purchased $1 \mu \mathrm{m}$ diameter $\mathrm{C} 18$ coated $\mathrm{SiO}_{2}$ spheres from Micromod company. $40 \mathrm{mg}$ of these spheres were mixed with $1 \mathrm{ml}$ of ethanol solvent and sonicated for 10 hours for complete miscibility. Silicon substrates were purchased from Siltronix (Intrinsic, orientation: 100). These substrates were cut into $10 \mathrm{~mm} \times 10 \mathrm{~mm}$ area samples. Then, they were sonicated in distilled water and ethanol for 30 minutes and treated with a plasma torch sequentially in order to remove any residual surface contamination and to increase the surface wettability.

This is an Open Access article distributed under the terms of the Creative Commons Attribution License 2.0, which permits unrestricted use, distribution, and reproduction in any medium, provided the original work is properly cited. 

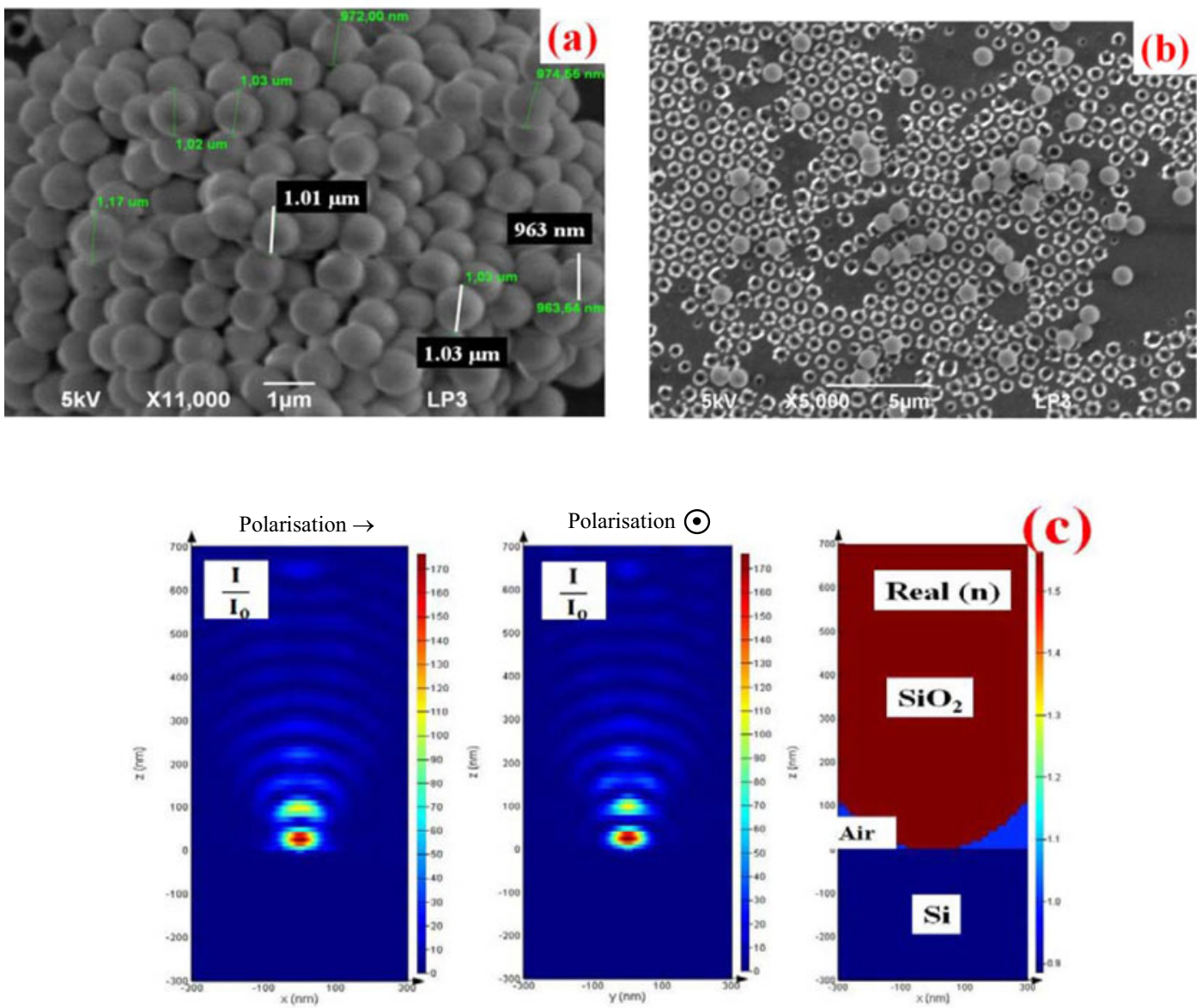

Figure 1. (a) $\mathrm{SEM}$ image of $\mathrm{C} 18$ functionalized $1 \mu \mathrm{m} \mathrm{SiO}$ particles (b) $\mathrm{SEM}$ image of nano-craters fabricated on $\mathrm{Si}$ substrate in single shot mode at the fluence of $0.55 \mathrm{~J} / \mathrm{cm}^{2}$. (c) FDTD calculations showing the cross sections of local fluence (or intensity) enhancement at the interface particle - substrate for polarized light illumination. I represents the intensity while $\mathrm{I}_{0}$ corresponds to the initial intensity of the beam along propagation (Z) axis. Schematic of the substrate and $\mathrm{SiO}_{2}$ are indicated in the right figure, along with the variation of real part of the refractive index $(\operatorname{Real}(\mathrm{n}))$.

Afterwards, we used Langmuir-Blodgett (LB) technique to grow monolayers of C18 functionalized $\mathrm{SiO}_{2}$ on $\mathrm{Si}$ substrates. $\mathrm{C} 18$ functionalized $\mathrm{SiO}_{2}$ spheres are hydrophobic and hence it is easier to grow monolayer using LB technique compared with bare $\mathrm{SiO}_{2}$ particles which are miscible in water. The use of LB technique in tandem with hydrophobic $\mathrm{SiO}_{2}$ particles resulted in good homogeneity of the monolayer over the entire substrates. In the past, we used spin-coating or drop-coating for the deposition technique for the self-assembly of spheres. Here the monolayer does not exhibit yet a good hexagonal close packed structure (hcp) but the total coverage of the substrate is ensured by the nature of the LB technique. This is a major asset for potential technological applications.

\section{EXPERIMENTAL RESULTS}

To irradiate the samples, we use an ArF excimer laser with top-hat beam profile at operating wavelength of $193 \mathrm{~nm}$ and delivering $0.5 \mathrm{~J}$ energy with pulse duration of $25 \mathrm{~ns}$. Repetition rate can be tuned from $1 \mathrm{~Hz}$ to $100 \mathrm{~Hz}$. A mask projection technique using $20 \mathrm{~cm}$ and $14 \mathrm{~cm}$ focal length lenses is implemented 

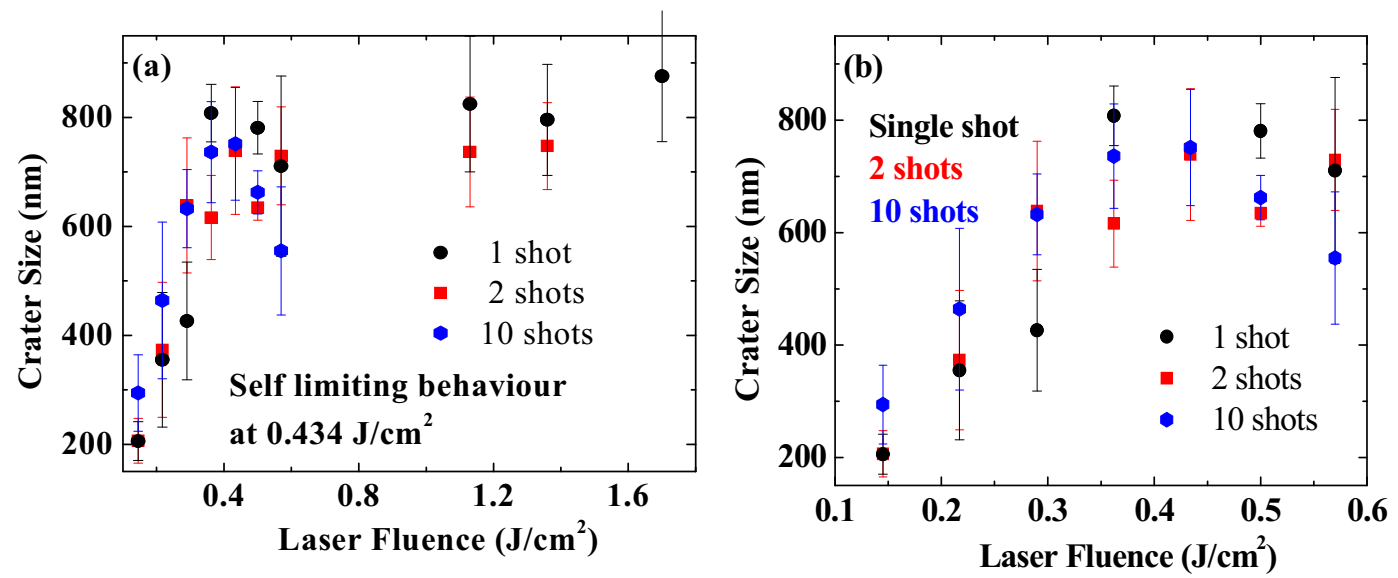

Figure 2. (a) Evolution of crater size with fluence in single, double and ten shots mode (b) Evolution of crater size with fluence in single, double and ten shots mode near the region where self-limiting behavior is observed.
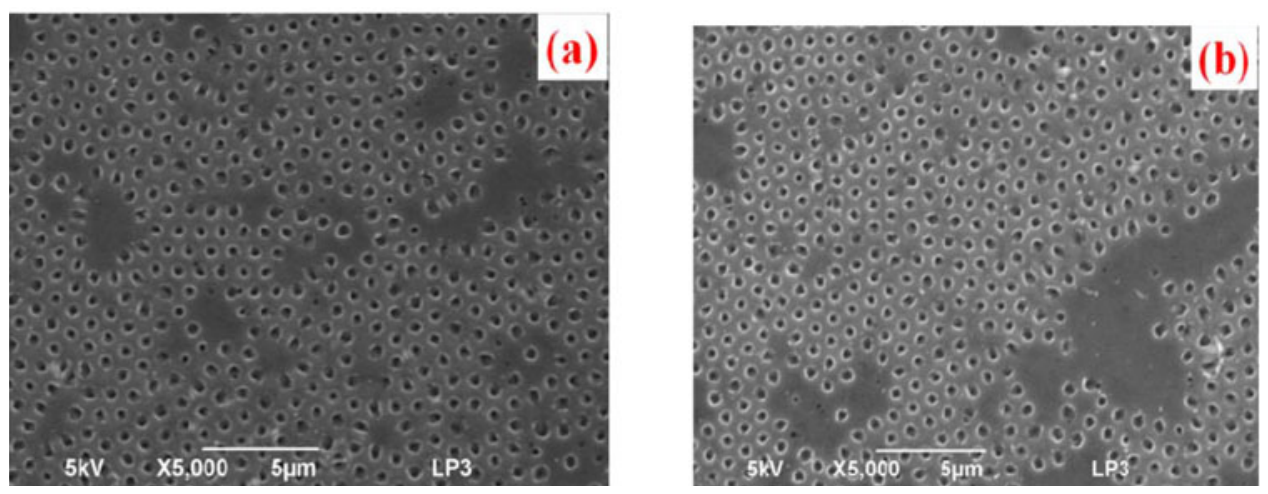

Figure 3. SEM image of nano craters fabricated at $0.55 \mathrm{~J} / \mathrm{cm}^{2}$ fluence (a) with two shots and (b) ten shots.

to achieve sufficient fluences on the target sample. An attenuator is set to vary the energy and thus the fluence onto the substrate. The substrate is placed on XY stages to change the location after each shot. We measured the energy after the lens to account for transmission losses for fluence calculations.

With the help of mask projection technique, lenses and attenuator, the fluence can be varied from 0.1 to $4.9 \mathrm{~J} / \mathrm{cm}^{2}$. In the following, only results till $1.7 \mathrm{~J} / \mathrm{cm}^{2}$ are discussed as strong damaging of the substrate occurs at high fluences. Figure 1a shows Scanning Electron Microscope (SEM) image of $1 \mu \mathrm{m}$ $\mathrm{SiO}_{2}$ agglomerated particles used in the experiments. The initial size dispersion of the particles in the films is roughly equal to $60 \mathrm{~nm}$ (standard deviation). The monolayer film is prepared on silicon substrate and is further irradiated using single, two shots and multi (5 and 10) shot modes. Figure $1 \mathrm{~b}$ shows the formation of nano-craters in single shot mode at the fluence of $0.55 \mathrm{~J} / \mathrm{cm}^{2}$. One can remark the variation in size of nano-craters fabricated, ranging from $650-800 \mathrm{~nm}$. This dispersion is mainly attributed to the initial size dispersion observed on the monolayer film yielding to different focusing conditions and strong fluence variation (amplified by the enhancement factor related to the micro lensing effect, as explained below) underneath the monolayer. Other sources of size dispersion result from effects such as local bilayers, and humidity. Any residual local humidity can for instance play an important role by changing the nature of the particle-substrate interface or eject the sphere by its explosive evaporation before substrate ablation, as established earlier [8,9]. A second interesting point is 


\section{Web of Conferences}
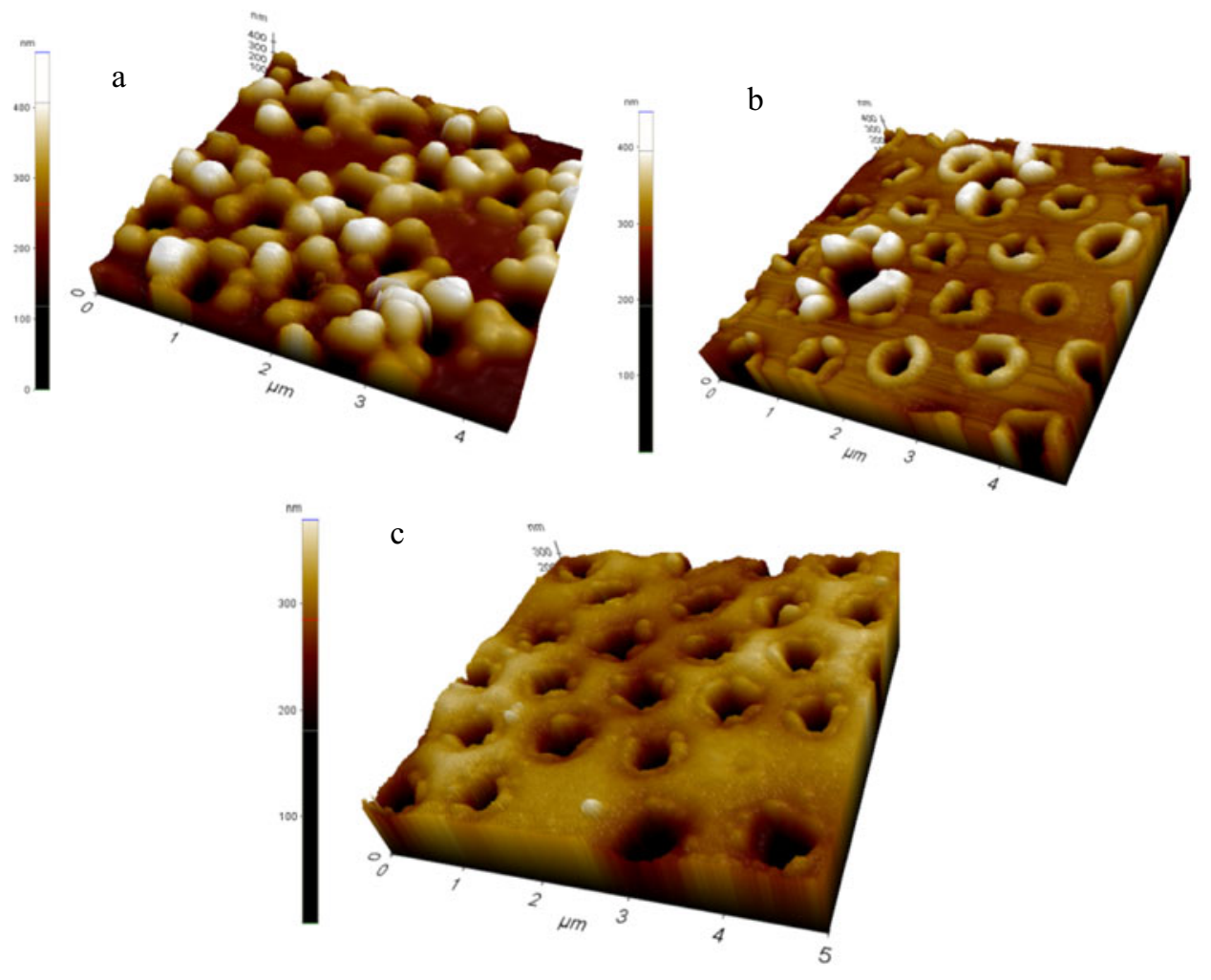

Figure 4. AFM Image of the surface morphology of Si substrate at the fluence of $0.55 \mathrm{~J} / \mathrm{cm}^{2}$ with (a) single shot (b) 2 shots and (c) 10 shots.

the observation of nano-craters on silicon though the fluence is well below the damage threshold of bare silicon, which is around $1 \mathrm{~J} / \mathrm{cm}^{2}$ with this laser [10]. This is due to the enhancement of fluence through photonic nanojet mechanism underneath the spheres $[1,11,12]$. We carried out FDTD calculations to predict the enhancement factor. Details on the calculation procedure can be found in [13]. The results are presented in Figure 1c. We note that the presence of the substrate is accompanied by a periodic modulation along the optical axis, revealing the interference between the incoming beam (see [4]) and its reflected part from silicon. In addition, we note the maximum intensity enhancement factor exceeds 160 inside the transparent sphere while it is of the order of 50 at the absorbing silicon surface. As a result of the enhanced fluence due to this micro lensing effect, a huge energy deposition takes place locally under the particle, yielding sphere removal and the formation of a crater with transverse dimensions smaller than the particle size.

Figure 2 shows the evolution of crater size with fluence and for different number of shots. Whatever the number of shots, we clearly see the increase in crater size till a threshold value around $0.45 \mathrm{~J} / \mathrm{cm}^{2}$. After the threshold value, there is no significant increment in the crater size further though fluence is increased for single and double shots (see figure 2a). This could be attributed to the self-limiting character of ablation experiment using particles as focusing element, as already reported in [13]. Indeed, since the particles are ejected by momentum transfer from the ablated species, the higher is the laser energy, the sooner is the particle detachment and the disappearance of the focusing power at the target surface. Then, increasing the laser fluence does not lead to an increase of the deposited energy and the crater size inside the substrate as it is commonly observed in experiments with conventional focusing optics. 
For ten shots, a decrease in size of the nano-craters is observed when the laser fluence is increased. We found that this behavior could be attributed to a strong annealing (or cleaning) occurring when using a large number of shots. In fact, considering irradiation in single shot mode, we observe heat affected zones and resettling of debris around the craters. However, as the number of shots increases, the crater morphology looks more homogenous and smooth. This is evidenced in Figure 3 showing SEM images of the nano-craters obtained at the fluence of $0.55 \mathrm{~J} / \mathrm{cm}^{2}$ with two and ten shots. When we compared with the figure $1 \mathrm{~b}$ for single shot, we conclude that increasing the number of shots results in cleaning or annealing the surface of the substrate. Further study and characterization are needed to fully understand the exact mechanism (annealing/cleaning or other effect) at play and yielding an improvement of the surface topography when using a large number of shots.

To confirm this aspect, we have further examined the quality of nano- and micro-craters obtained with an Atomic Force Microscope (AFM). Figure 4a-c shows the surface morphologies of the nanocraters in single, double and ten shot modes at the fluence of $0.55 \mathrm{~J} / \mathrm{cm}^{2}$. From these AFM images, it is evident that the surface morphology becomes smoother when increasing the number of shots. In case of Figure $4 \mathrm{a}$ (single shot), ablated and heat affected zones are clearly visible as bubbles on the surface. In case of two shots as shown in figure $4 \mathrm{~b}$, the surface morphology is smoother compared with single shot mode though there are still some bubbles appear on the surface. In case of ten shots (Figure 4c), almost no bubbles appear on the surface. This confirms our assumption that the surface becomes smoother and regular with the number of shots. The depth and diameter of these craters for ten shots at the fluence of $0.55 \mathrm{~J} / \mathrm{cm}^{2}$ were measured at around $230 \mathrm{~nm}$ to $400 \mathrm{~nm}$ using AFM.

\section{CONCLUSIONS}

In this paper, we presented our results on the fabrication of high density nano-craters on silicon substrate by UV nanosecond laser ablation and using the LB technique to prepare a near-field focusing optical structure. We showed the role of micro lensing effect by using low fluences to fabricate craters on silicon through photonic nanojet assisted mechanism. In addition, the results on crater formation exhibit a self-limiting character which maintains the size of craters at the sub-micrometer scale for all laser fluences. Well-defined array of craters and of good quality (surface regularity and smoothness) were obtained at modest fluences and large number of shots. Surface cleaning and/or annealing (or another effect remaining to be identified in the near future) taking place with multi-shots can be used as a tool to obtain clear and smooth craters, as evidenced by electron and AFM microscopy.

\section{Acknowledgements}

We thank French ANR agency for financial support through the FELINS-ANR-10-BLAN-946 project.

\section{References}

[1] A. V. Itagi, W. A. Challener, J. Opt. Soc. Am. A. 22, 2847 (2005)

[2] P. Jiang, Tushar Prasad, Michael J. McFarland, Vicki L. Colvin, Appl. Phys. Lett. 89, 011908 (2006)

[3] S. H. Sun, C. B. Murray, Dieter Weller, Lies Folks, Andreas Moser, Science 287, 1989 (2000)

[4] A. Pereira, D. Grojo , M. Chaker, P. Delaporte, D. Guay, M. Sentis, Small 4, 572 (2008)

[5] J. Wenger, D. Gerard, H. Aouani, H. Rigneault, Anal. Chem. 80, 6800 (2008)

[6] J. J. Schwartz, S. Stavrakis, S. R. Quake, Nat. Nanotechnol. 5, 127 (2010)

[7] Y. Zhou, M. H. Hong, F. Fuh, L. Lu, B. S. Luk'yanchuck, Z. B.Wang, L. P. Shi, T. C. Chong, App. Phys. Lett. 88, 023110 (2006)

[8] D. Grojo, P. Delaporte, M. Sentis, O. H. Pakarinen, and A. S. Foster, Appl. Phys. Lett. 92, 033108 (2008) 


\section{Web of Conferences}

[9] D. Grojo, P. Delaporte, M. Sentis, J. Adh. Sci. Tech. 22, 623 (2008)

[10] D. Grojo, A. Cros, P. Delaporte, M. Sentis, Appl. Surf. Sci. 253, 8309 (2007)

[11] P. Kuhler, F. J. Garcia de Abajo, J. Solis, M. Mosbacher, P. Leiderer, C. N. Afonso, J. Siegel, Small 5, 1825 (2009)

[12] Sylvain Lecier, Yoshitate Takakura, Patrick Meyrueis, Opt. Lett. 30, 2641 (2005)

[13] D. Grojo, L. Charmasson, A. Pereira, M. Sentis, P. Delaporte, J. Nanosci. Nanotechnol. 11, 9129 (2011) 